\title{
A 160-GHz Frequency-Translation Phase-Locked Loop With RSSI Assisted Frequency Acquisition
}

\author{
Wei-Zen Chen, Tai-You Lu, Yan-Ting Wang, Jhong-Ting Jian, Yi-Hung Yang, and Kai-Ting Chang
}

\begin{abstract}
A 160-GHz frequency-translation PLL with tuning range from $156.4 \mathrm{GHz}$ to $159.2 \mathrm{GHz}$ is presented. Sub- THz 1/9 prescaler is replaced by a 3rd harmonic mixer incorporating a frequency tripler for frequency down conversion. A transformer-based VCO is utilized to alleviate capacitive and resistive load associated with varactor and succeeding buffer stages. Frequency acquisition is assisted by received signal strength indicator (RSSI) for automatic frequency sweeping and fast locking. Fabricated in $65 \mathrm{~nm}$ CMOS technology, the chip size is $0.92 \mathrm{~mm}^{2}$. The PLL locking time is less than $3 \mu \mathrm{s}$. This chip drains $24 \mathrm{~mW}$ from a $1.2 \mathrm{~V}$ power supply.
\end{abstract}

Index Terms-Harmonic mixer, PLL, RSSI, tripler.

\section{INTRODUCTION}

C MOS Terahertz $(\mathrm{THz})$ systems are considered as emerging technology for advanced imaging and spectroscopy for sensing and detection applications, and have drawn tremendous research efforts recently [1], [2]. As limited by the unity maximum available power gain frequency of transistor $\left(f_{\max }\right)$, CMOS THz signal generators rely on the harmonic generation of a sub-THz reference using phase combination or push-push techniques [3], [4]. To implement a high speed phase-locked loop, multi-stage cascaded injection-locked frequency dividers (ILFD) [5]-[7] are commonly used in the feedback path to down covert the VCO output frequency for phase comparison. For the oscillation frequency close to hundreds of $\mathrm{GHz}$ range, the frequency tuning ranges of both VCO and ILFD become very limited, which are highly susceptible to parasitic effects associated with the buffer stage and interconnects. As the ILFD is prone to self-oscillation without properly injection locked, frequency misalignments in feedback chain are susceptible to PVT variations and may cause the loop fail to lock. To circumvent the critical issue, this paper proposes a novel frequency-translation PLL (FT-PLL) for over $100 \mathrm{GHz}$ operations.

In the proposed FT-PLL, high speed prescalers are replaced by a harmonic mixer incorporating frequency tripler to down convert VCO frequency from sub-THz to around $1 \mathrm{GHz}$, which

Manuscript received May 05, 2013; revised October 03, 2013; accepted November 12, 2013. Date of publication January 29, 2014; date of current version May 23, 2014. This work is supported in part by project NSC-100-2220-E-009-011, and TSMC University Shuttle Program for the fabrication of this design. This paper was recommended by Associate Editor J. Kim.

W.-Z. Chen is with the Department of Electronics Engineering, National Chiao-Tung University, Hsin-Chu 30010, Taiwan (e-mail: wzchen@mail.nctu.edu.tw).

T.-Y. Lu is with MediaTek Inc., Hsin-Chu 30078, Taiwan.

J.-T. Jian is with Sitronix Technology Corporation, Taipei 11444, Taiwan.

Y.-T. Wang, Y.-H. Yang, and K.-T. Chang are with National Chiao-Tung University, Hsin-Chu 30010, Taiwan.

Color versions of one or more of the figures in this paper are available online at http://ieeexplore.ieee.org.

Digital Object Identifier 10.1109/TCSI.2013.2295016

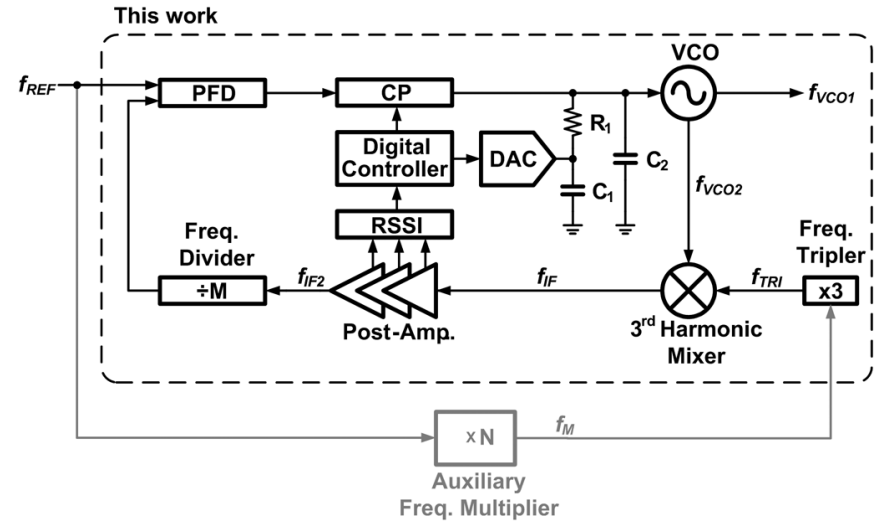

Fig. 1. FT-PLL architecture.

can be easily scaled down by digital divider. Besides, a received signal strength indicator (RSSI) is used for automatic frequency sweeping to overcome PVT variations and extend locking range.

This paper is organized as follows. Section II describes the proposed FT-PLL architecture and operation principle. The system behavior is discussed in Section III. Section IV describes the detailed circuit schematic. Experimental results are shown in Section V, and Section VI concludes this paper.

\section{ARCHITECTURE}

Fig. 1 depicts the proposed FT-PLL architecture. The sub-THz output frequency $\left(f_{V C O}\right)$ is generated from an on-chip LC oscillator. To down convert $f_{V C O}$ for phase comparison, an auxiliary frequency multiplier generates $f_{M}$ $\left(\mathrm{N} f_{R E F}\right)$, whose output frequency is then tripled $\left(f_{T R I}\right)$ by an on chip frequency tripler to drive a 3rd harmonic mixer. Through harmonic mixing with $f_{T R I}, f_{V C O}$ is translated to a much lower frequency band $\left(f_{I F}\right)$ [8], and then enlarged by a post amplifier to drive a digital frequency divider (1/M). When the loop is locked, we have

$$
\begin{gathered}
f_{T R I}=3 f_{M}=3 N f_{R E F} \\
f_{V C O}-3 f_{T R I}=M_{R E F} \\
f_{V C O}=(9 N+M) f_{R E F} .
\end{gathered}
$$

It can be seen that the frequency tripler incorporating with 3rd harmonic mixer performs the same function as a $1 / 9$ prescaler, and $f_{V C O}$ can be adjusted by programming the divide ratio $\mathrm{M}$ or the frequency multiplication factor $\mathrm{N}$. To alleviate bandwidth requirement of frequency tripler, $f_{M}$ is fixed in this design. It also facilitates the optimization of frequency tripler and harmonic mixer. Besides, the bandwidth of post amplifier is designed to cover the frequency tuning range of $M f_{R E F}$. It en- 


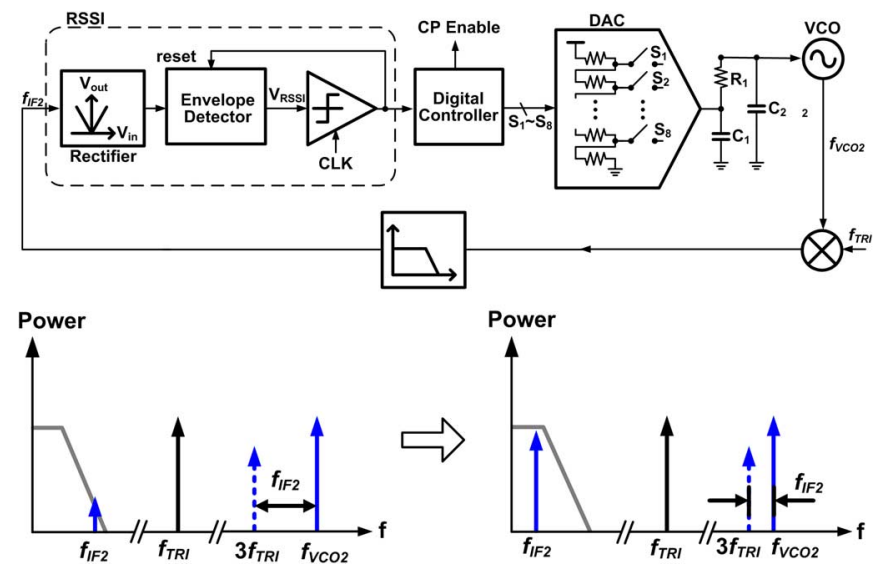

Fig. 2. PLL with RSSI assisted frequency tracking scheme.

larges the signal swing of $f_{I F}$ to logic level for a proper operation of digital divider.

Using a mixer for frequency down conversion, the alternative solution of $f_{I F}$ is at $3 f_{T R I}-f_{V C O}$. To avoid this ambiguity, the oscillator free running frequency is preset to its highest frequency at the onset of frequency switching, which is higher than $3 f_{T R I}$ to guarantee a single mode operation.

The procedure for phase and frequency locking is described as follows. When the channel switching command is issued, the VCO is preset to its highest frequency controlled by a 3 bits resistor ladder DAC, and the PLL is opened loop by disabling the charge pump current. Meanwhile, the received signal strength indicator (RSSI) continues monitoring the power level at $f_{I F 2}$. Fig. 2 illustrates the frequency acquisition scheme incorporating RSSI. The harmonic mixer output is fed into a post voltage amplifier, and the RSSI is composed of a rectifier followed by an envelope detector and a comparator. The comparator output is stored in a digital controller to adjust the resistor ladder DAC. Once the DAC output is renewed, the envelope detector will be reset to track the new signal amplitude at $f_{I F 2}$. Meanwhile, the VCO frequency $f_{V C O}$ is adjusted by charging the loop filter capacitor $\mathrm{C}_{1}$ through DAC. By the low-pass nature of the post voltage amplifier, for $f_{V C O} \gg 3 f_{T R I}$, a higher $f_{I F}$ leads to a smaller received signal amplitude. According to RSSI, the digital controller then updates the DAC contents in a descending way to reduce $f_{V C O}$, pulling in the frequency gradually to approach $3 f_{T R I}$. As the $f_{I F}$ falls into the bandwidth of post amplifier, its signal amplitude would become sufficiently larger to toggle the post frequency divider properly, as is illustrated in Fig. 2.

Moderated by the RSSI and level detector, this pull-in process will be finished when the signal amplitude at $f_{I F 2}$ exceeds the threshold level to drive the succeeding frequency divider. In this design, the input sensitivity of the post divider is around 150 $\mathrm{mV}$, thus the threshold voltage of comparator is set to $180 \mathrm{mV}$ to tolerate $20 \%$ PVT variation. The 3 bits DAC provides voltage tuning range of $0.9 \mathrm{~V}$ and corresponding frequency tuning range of $3 \mathrm{GHz}$. As the DAC is updated at $4 \mathrm{MHz}$, the frequency sweeping time is less than $2 \mu \mathrm{s}$. After the pull-in process monitored by RSSI, the digital controller then resumes the close loop operation by enabling the charge pump current, and keeps $f_{V C O}$ phase tracking $f_{R E F}$. The proposed frequency sweeping scheme brings $f_{V C O}$ into its target frequency automatically, alleviating bandwidth requirement of the feedback path of PLL without sacrificing its locking range.

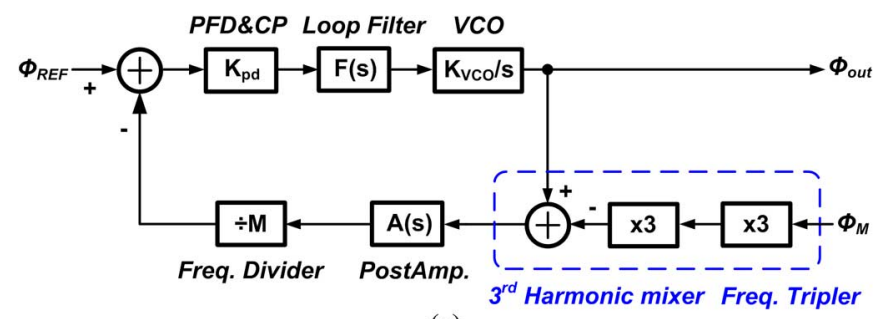

(a)

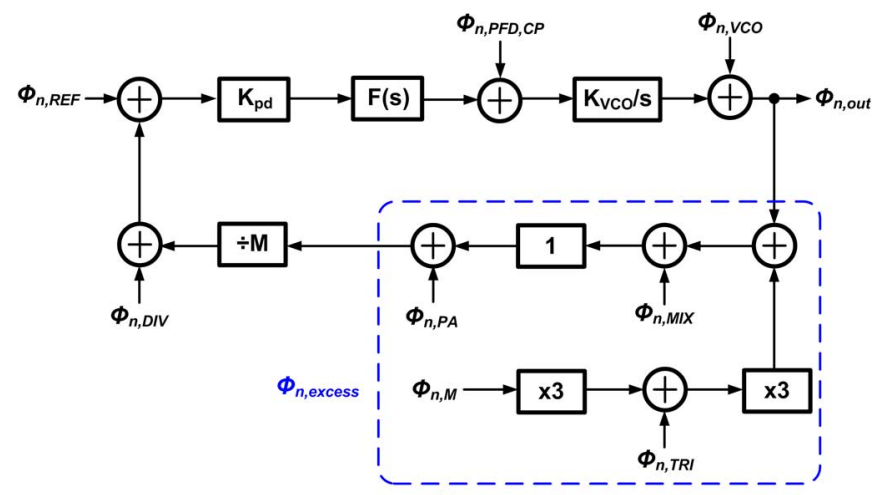

(b)

Fig. 3. (a) Frequency-translation PLL behavioral model (b) noise model.

\section{BehaVioral Model}

To investigate behavior and phase noise performance of the proposed FT-PLL, its linear model is illustrated in Fig. 3(a). Here $K_{p d}$ represents the gain of phase frequency detector (PFD) and charge pump (CP), $K_{V C O}$ denotes $\mathrm{VCO}$ gain, $F(s)$ and $A(s)$ respectively represent the transfer function of loop filter and post amplifier. The linear model of $3 \mathrm{rd}$ harmonic mixer and frequency tripler is illustrated in the dashed box, which provides phase offset of $9 \phi_{M}\left(\mathrm{~N} \phi_{R E F}\right)$ in the feedback path. The phase transfer function of $\phi_{R E F}$ to $\phi_{\text {out }}$ can be derived as

$$
\frac{\phi_{\text {out }}}{\phi_{\text {REF }}}=\frac{\left[1+\frac{9 N A(s)}{M}\right] G(s)}{1+\frac{A(s) G(s)}{M}}
$$

where $G(s)=K_{p d} K_{V C O} F(s) / s$. When the PLL is operating in phase tracking mode, $f_{I F}$ falls within the post amplifier's -3 $\mathrm{dB}$ bandwidth, and the phase shift caused by $A(s)$ is negligible $(A(s) \approx 1)$. Thus the system transfer function can be simplified as

$$
\frac{\phi_{\text {out }}}{\phi_{\text {REF }}}=\left.\frac{\left[1+\frac{9 N A(s)}{M}\right] G(s)}{1+\frac{A(s) G(s)}{M}}\right|_{G(s) \rightarrow \infty}=9 N+M
$$

which is the same as that of a conventional PLL with frequency multiplication factor of $9 N+M$. Fig. 3(b) illustrates the phase noise contribution from individual building blocks, where, $\phi_{n, R E F}, \phi_{n, P F D, C P}, \phi_{n, V C O}$ and $\phi_{n, D I V}$ respectively represent the noise source from reference input, loop filter, VCO, and frequency divider in a typical charge-pump based PLL. Their impacts on the VCO phase noise $\left(\phi_{n, \text { out }}\right)$ can be respectively represented as

$$
H_{1}(s) \equiv \frac{\phi_{n, o u t 1}}{\phi_{n, R E F}}=\frac{G(s)}{1+\frac{G(s)}{M}}
$$




$$
\begin{aligned}
& H_{2}(s) \equiv \frac{\phi_{n, \text { out } 2}}{\phi_{n, P F D, C P}}=\frac{\frac{1}{s} K_{V C O}}{1+\frac{G(s)}{M}} \\
& H_{3}(s) \equiv \frac{\phi_{n, \text { out } 3}}{\phi_{n, \text { VCO }}}=\frac{1}{1+\frac{G(s)}{M}} \\
& H_{4}(s) \equiv \frac{\phi_{n, \text { out } 4}}{\phi_{n, D I V}}=\frac{G(s)}{1+\frac{G(s)}{M}}
\end{aligned}
$$

Assuming that the frequency multiplier output $f_{M}$ is generated from an auxiliary PLL, its phase noise $\left(\phi_{n, M}\right)$ can be described as

$$
\phi_{n, M} \equiv H_{a}(s) \phi_{n, R E F}+\phi_{n, A u x P L L}
$$

where $H_{a}(s)$ represents the reference phase noise transfer function (low pass filtering) of the auxiliary PLL, whose DC gain equals to $N$ (frequency multiplying factor), and $\phi_{n, A u x P L L}$ denotes other noise sources coming from the auxiliary PLL. Defining the excess noise source in the frequency-translation PLL (FT-PLL) as $\phi_{n, \text { excess }}$, where

$$
\begin{aligned}
\phi_{n, \text { excess }}= & 9 \phi_{n, M}+3 \phi_{n, T R I}+\phi_{n, M I X}+\phi_{n, P A} \\
= & 9 H_{a}(s) \phi_{n, R E F}+9 \phi_{n, A u x P L L}+3 \phi_{n, T R I} \\
& +\phi_{n, M I X}+\phi_{n, P A} .
\end{aligned}
$$

We have

$$
H_{5}(s) \equiv \frac{\phi_{n, o u t 5}}{\phi_{n, \text { access }}}=\frac{\frac{G(s)}{M}}{1+\frac{G(s)}{M}} .
$$

Combing (6) and (12), it reveals that the reference phase noise transfer function $H_{R E F}(s)$ can be described as

$$
H_{R E F}(s)=9 H_{a}(s) H_{5}(s)+H_{1}(s)
$$

whose DC gain equals to $(9 N+M)$. The 1 st term corresponding to $9 N$ is noise shaped by the cascaded loop filter $H_{a}(s) H_{5}(s)$, which facilitates optimization compared to a single loop architecture [9]. Meanwhile, the other terms in $\phi_{n, \text { excess }}\left(9 \phi_{n, A u x P L L}+3 \phi_{n, T R I+} \phi_{n, M I X+} \phi_{n, P A}\right)$ are translated to VCO output as in-band noise without amplification. According to (7)-(9), the noise contribution from $\phi_{n, C P}$ and $\phi_{n, D I V}$ in the main loop is reduced from $(9 N+M)$ to $(M)$ in contrast to a single loop PLL. Additionally, in-band noise from VCO is suppressed by increasing the loop gain (feedback factor increases by $(9 \mathrm{~N}+\mathrm{M}) / \mathrm{M}$ compared to single loop PLL). It provides some design margin to accommodate noise degradation caused by $\phi_{n, \text { excess }}$.

To sum up, the FT-PLL output phase noise $\phi_{n, \text { out }}$, can be expressed as

$$
\overline{\phi_{n, \text { out }}^{2}}=\overline{\phi_{n, \text { out } 1}^{2}}+\overline{\phi_{n, \text { out } 2}^{2}}+\overline{\phi_{n, \text { out } 3}^{2}}+\overline{\phi_{n, \text { out } 4}^{2}}+\overline{\phi_{n, \text { out } 5}^{2}}
$$

Provided that the reference noise floor is $-150 \mathrm{dBc} / \mathrm{Hz}$ at $1 \mathrm{MHz}$ offset, $\mathrm{M}=8$ and $\mathrm{N}=134$, the loop bandwidth is chosen where the out-of-band noise and the loop noise contribute equally to the output jitter, the simulated in band phase noise is about $-89 \mathrm{dBc} / \mathrm{Hz}$.

\section{BUILDING BLOCKS}

Design considerations of the individual building blocks are described as follows.

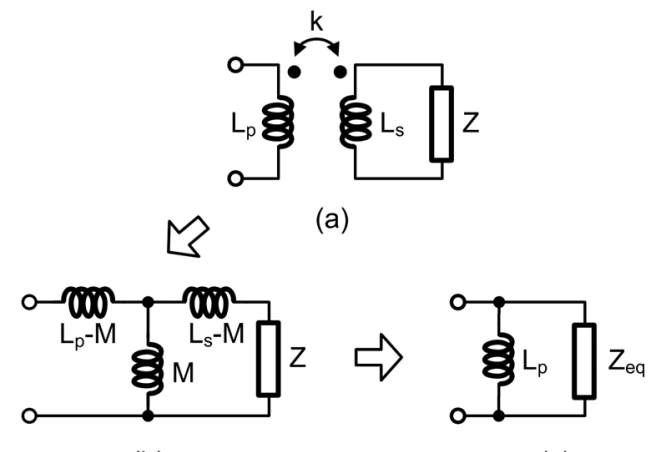

(b)

(c)

Fig. 4. The equivalent model of transformer.

\section{A. Voltage Controlled Oscillator}

As $f_{V C O}$ approaches devices' $\mathrm{f}_{\max }$, the parasitic resistance introduced by the LC tank and its succeeding buffer stages becomes a critical issue to maintain stable oscillation. Meanwhile, the parasitic capacitance severely limits the oscillation frequency of VCO. To overcome the design challenges, a transformer-based VCO is adopted.

To demonstrate the advantage of capacitive degeneration through magnetic coupling, Fig. 4(a) exemplifies a two-coil model for simplicity [10]. For a two-coil transformer with primary coil inductance of $L_{p}$, secondary coil inductance of $L_{s}$, mutual inductance of $\mathrm{M}$, and coupling factor of $k$, its equivalent T-model can be depicted as shown in Fig. 4(b). When the secondary port is loaded with impedance $(Z)$, the equivalent impedance $\left(Z_{e q}\right)$ seen from the primary port and paralleled with $L_{p}$, as shown in Fig. 4(c), becomes

$$
Z_{e q}=\frac{1}{k^{2}}\left[\frac{L_{p}}{L_{s}} Z+j \omega L_{p}\left(1-k^{2}\right)\right]
$$

By choosing $\left(L_{p} / k^{2} L_{s}>1\right)$, the loading effect associated with the secondary port can be reduced accordingly.

Fig. 5 illustrates the detail circuit schematic. The VCO core is composed of negative impedance converter $\left(M_{1}-M_{2}\right)$ and the primary coil $\left(L_{p}\right)$ of the transformer, while the varactors, buffer stage, and harmonic mixer are coupled to the resonator through an outer coil $\left(L_{s 1}\right)$ and inner coil $\left(L_{s 2}\right)$ respectively. As

$$
L_{s 1}<L_{p}<L_{s 2}
$$

it also degenerates the tuning sensitivity of the varactor to maintain a high frequency oscillation. Meanwhile, the transformer coupling also provides voltage gain between the primary and secondary ports, where

$$
\frac{v_{o}}{v_{i}}=k \sqrt{\frac{L_{s}}{L_{p}}} .
$$

It provides the advantage to generate a higher voltage swing at the input of 3rd harmonic mixer. The detail layout of the triple-coil transformer and its parameters are also summarized in Fig. 5.

The transformer is laid out as micro strip lines using top metal, providing inductance of about $27.5 \mathrm{pH}$ and self-resonant frequency of about $372 \mathrm{GHz}$. The coupling coefficients from the primary coil to the secondary and the third coil are about 0.103 

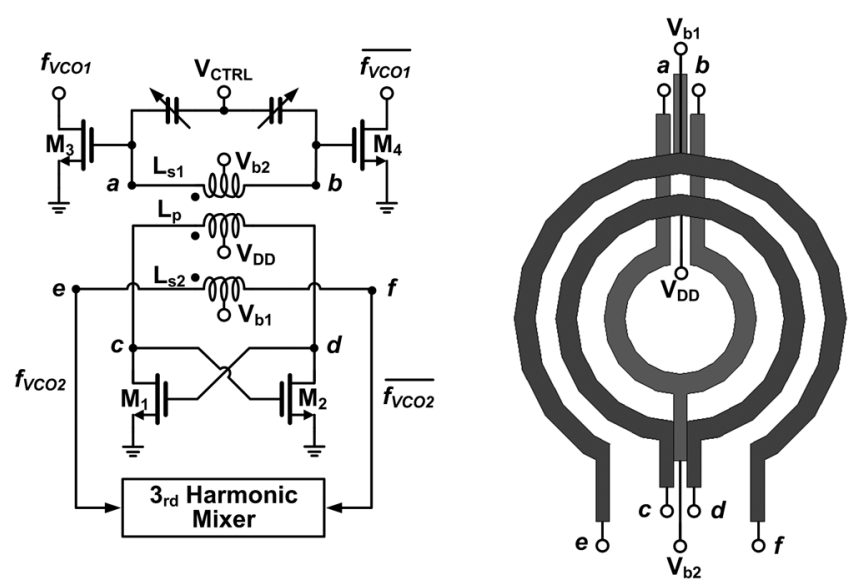

\begin{tabular}{|l|c|c|c|}
\hline & Width $(\mu \mathrm{m})$ & Inductance $(\mathrm{pH})$ & $f_{\text {self-resonant }}(\mathrm{GHz})$ \\
\hline $\mathrm{L}_{\mathrm{p}}$ & 3 & 27.49 & 372.4 \\
\hline $\mathrm{L}_{\mathrm{s} 1}$ & 3 & 22.32 & 399.3 \\
\hline $\mathrm{L}_{\mathrm{s} 2}$ & 3 & 42.57 & 375.6 \\
\hline
\end{tabular}

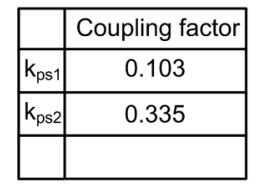

Fig. 5. VCO schematic.

and 0.335 respectively. By impedance transformation, the parasitic capacitance and resistance seen at the resonator are about $13 \mathrm{fF}$ and $243 \Omega$ as referred to the primary coil. For a varactor tuning range from $6.7 \mathrm{fF}$ to $16.6 \mathrm{fF}$, the measured tuning range of the $\mathrm{VCO}$ is from $156.4 \mathrm{GHz}$ to $159.2 \mathrm{GHz}$.

\section{B. Frequency Tripler}

Fig. 6 illustrates the circuit schematic of frequency tripler. The frequency tripler [11] is configured as a synchronous lock oscillator composing of $M_{3}, M_{4}$, and LC tank, whose output frequency $\left(f_{T R I}\right)$ is tuned at $3 N f_{R E F}$. The excitation signal $\left(f_{M}\right)$ provided by the auxiliary frequency multiplier is injected differentially at $\mathrm{M}_{1}$ and $\mathrm{M}_{2}$ to generate the third harmonic tone of $f_{M}$, which is provided to synchronize the injection locked oscillator. Since both the $f_{M}$ and $f_{T R I}$ frequencies are fixed, it facilitates the optimization of the tripler design with maximum power gain by properly choosing the resonant frequency of the tank. As the locking range of the frequency tripler can be described as [12]

$$
\omega_{L}=\frac{\omega_{0}}{2 Q} \frac{I_{3}}{I_{o s c}}
$$

where $\omega_{0}$ and $Q$ represent the center frequency and quality factor of the LC tank, $I_{3}$ represents the third harmonic drain current of $\mathrm{M}_{1}$ and $\mathrm{M}_{2}$, and $I_{\text {osc }}$ represents the DC current of the injection locked oscillator. The 3rd harmonic of the transconductance of $\mathrm{M}_{1}$ and $\mathrm{M}_{2}$ can be maximized to enhance the injection efficiency by properly choosing their conduction angle $(\phi)$ [13]. The strength of the third harmonic component under different conduction angle can be evaluated by normalizing its amplitude $I_{3}$ to that of the overall drain current $I_{\max }$, where

$$
\frac{I_{3}}{I_{\max }}=\frac{\sin \left(\frac{3 \phi}{2}\right) \cos \left(\frac{\phi}{2}\right)-3 \cos \left(\frac{3 \phi}{2}\right) \sin \left(\frac{\phi}{2}\right)}{12 \pi\left(1-\cos \frac{\phi}{2}\right)} .
$$

As shown in Fig. 7, the frequency tripler has the highest injection efficiency at the conduction angle of about $80^{\circ}$. Under this

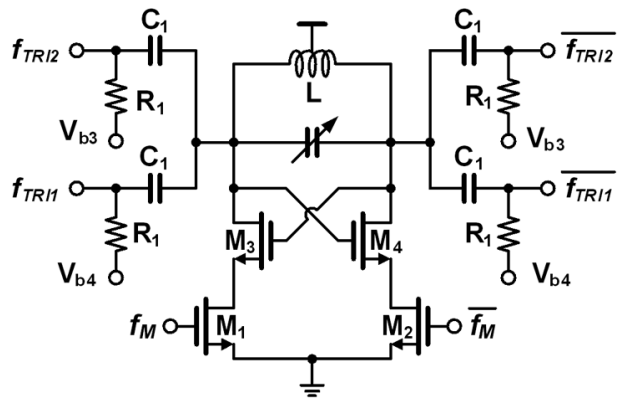

Fig. 6. Frequency tripler schematic.

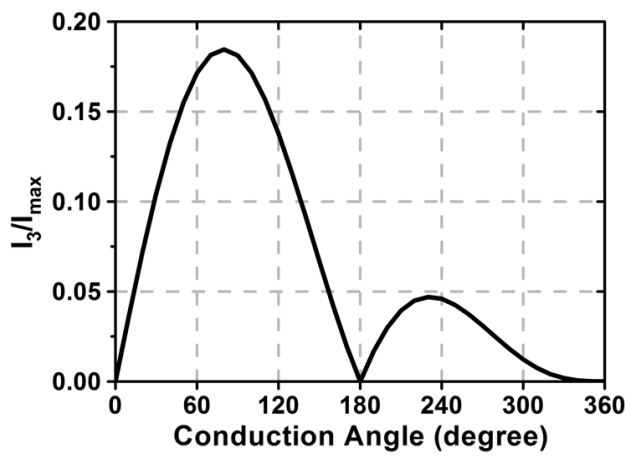

Fig. 7. Injection efficiency of frequency tripler under different conduction angle.

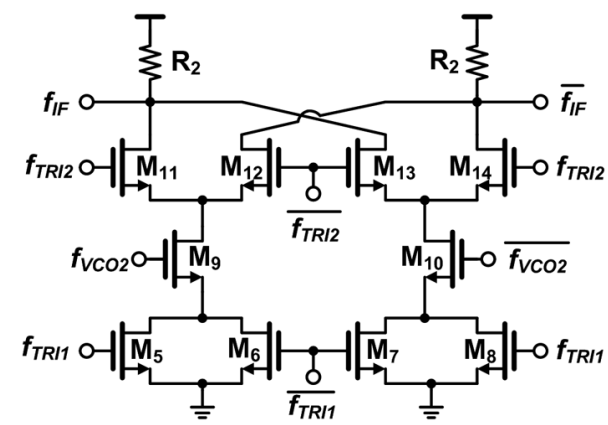

Fig. 8. 3rd harmonic mixer schematic.

circumstance, $\mathrm{M}_{1}$ and $\mathrm{M}_{2}$ are biased at sub-threshold region, which may cause the oscillator fail to start oscillation due to limited DC current. To overcome this issue, a conduction angle of about $240^{\circ}$ is chosen in this design for a higher injection efficiency while meeting the oscillation criterion.

\section{3rd Harmonic Mixer}

Fig. 8 illustrates the circuit schematic of $3 \mathrm{rd}$ harmonic mixer. It is composed of frequency doubler $\left(M_{5}-M_{8}\right)$, a single transistor mixer $\left(M_{9}-M_{10}\right)$, and a double-balanced Gilbert switches $\left(M_{11}-M_{14}\right)$ in cascode to reduce power consumption. $f_{T R I}$ is injected differentially to the transistor $\left(M_{5}-M_{8}\right)$ and $\left(M_{11}-M_{14}\right)$, and the VCO output is applied to $\left(M_{9}-M_{10}\right)$ as $f_{V C O 2}$.

Let

$$
f_{T R I}=A_{T R I}\left(\cos \omega_{T R I} t\right)
$$

and

$$
f_{V C O 2}=A_{V C O}\left(\cos \omega_{V C O} t\right) .
$$


The drain current of $M_{5}-M_{8}$ can be expressed as [14]

$i_{D 5-8} \approx\left(\mu_{n} C_{o x} \frac{W}{L}\right)_{5-8}\left(V_{O V 5-8}^{2}+f_{T R I}^{2} \pm 2 V_{O V 5-8} f_{T R I}\right)$

where $V_{O V 5-8}$ are the overdrive voltages of $M_{5}-M_{8}$.

By connecting the output currents of $\mathrm{M}_{5}, \mathrm{M}_{6}$, and $\mathrm{M}_{7}, \mathrm{M}_{8}$, the source voltage of $M_{9}$ and $M_{10}$ can be approximated as

$$
\begin{aligned}
v_{s 9} & =v_{s 10} \approx\left(i_{D 5}+i_{D 6}\right) R_{L} \\
& \approx \frac{1}{2}\left(\mu_{n} C_{o x} \frac{W}{L}\right)_{5-8} A_{T R I}^{2} R_{L} \cos 2 \omega_{T R I} t+V_{D C}
\end{aligned}
$$

where $R_{L}$ represents the effective impedance at the source node of $M_{9}$ and $M_{10}$, and $V_{D C}$ denotes the DC voltage.

From (23), it can be seen that the odd order harmonics of $f_{T R I}$ are cancelled out at the source of $\mathrm{M}_{9}$ and $\mathrm{M}_{10}$, while its even order harmonics remain. To maximize the second order harmonic tone of the frequency doubler, $M_{5}$ to $M_{8}$ are biased at subthreshold region to perform as ideal switches and boost the harmonic tones.

As the overdrive voltage of $\mathrm{M}_{9}$ and $\mathrm{M}_{10}$ are modulated by the second order harmonic of $f_{T R I}$ and $f_{V C O 2}$, they perform as single transistor mixers through the nonlinearity of MOSFET I-V characteristic. Their output currents become

$$
\begin{gathered}
i_{d 9,10} \approx \frac{A_{1}}{2} \cos \left(2 \omega_{T R I}-\omega_{V C O 2}\right) t+A_{2} \cos \omega_{V C O 2} t+ \\
A_{3} \cos 2 \omega_{T R I} t+\frac{A_{1}}{2} \cos \left(2 \omega_{T R I}+\omega_{V C O 2}\right) t+\cdots
\end{gathered}
$$

where

$$
A_{1}=\left(\frac{\mu_{n} C_{o x}}{2} \frac{W}{L}\right)_{5-8}\left(\mu_{n} C_{o x} \frac{W}{L}\right)_{9-10} A_{V C O} A_{T R I}^{2} R_{L} .
$$

The other high frequency terms due to leakage $\left(A_{2}, A_{3}\right)$ and harmonic tones are low pass filtered at the input of Gilbert switches. Thus the drain currents of $\mathrm{M}_{9}$ and $\mathrm{M}_{10}$ are translated to $\left(f_{V C O}-2 f_{T R I}\right)$ and flow through the commutating stages $M_{11}-M_{14}$ driven by $f_{T R I}$ again.

The resulting down converted signal $f_{I F}$ is at $\left(f_{V C O 2}-\right.$ $\left.3 f_{T R I}\right)$, and the unwanted spurs at $\left(f_{V C O 2}-f_{T R I}\right)$ and $\left(f_{V C O 2}+3 f_{T R I}\right)$ are also filtered out by the RC low pass filter. If $\mathrm{M}_{11}-\mathrm{M}_{14}$ is modeled as ideal switches, the conversion gain $\left(G_{\text {Mixer }}\right)$ of the third harmonic mixer can be derived as

$$
\begin{aligned}
G_{\text {Mixer }} & \approx \frac{2}{\pi} \frac{A_{1}}{A_{V C O}} R_{2} \\
& =\frac{1}{\pi}\left(\mu_{n} C_{o x} \frac{W}{L}\right)_{5-8}\left(\mu_{n} C_{o x} \frac{W}{L}\right)_{9-10} A_{T R I}^{2} R_{L} R_{2} .
\end{aligned}
$$

If

$$
R_{L} \approx \frac{1}{g_{m 9-10}}=\frac{1}{V_{o v 9-10}\left(\mu_{n} C_{o x} \frac{W}{L}\right)_{9-10}} .
$$

Combing (26) and (27), we have

$$
G_{\text {mixer }} \propto\left(\mu_{n} C_{o x} \frac{W}{L}\right)_{5-8} \frac{1}{V_{o v, 9-10}} A_{T R I}^{2} R_{2} .
$$

According to (28), $G_{M i x e r}$ can be enhanced by increasing $A_{T R I}$ and decreasing the overdrive voltage of $\mathrm{M}_{9}$ and $\mathrm{M}_{10}$,

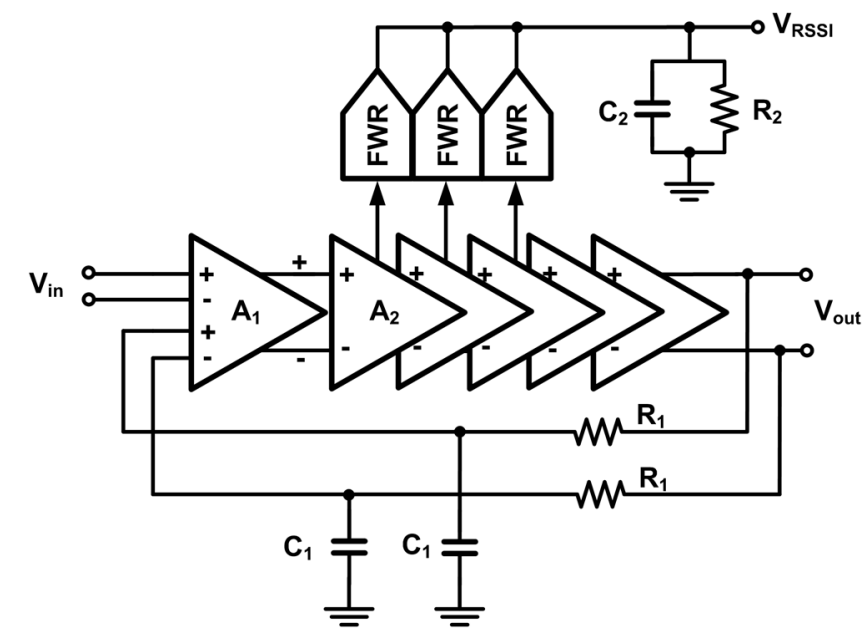

Fig. 9. Post-amplifier schematic.

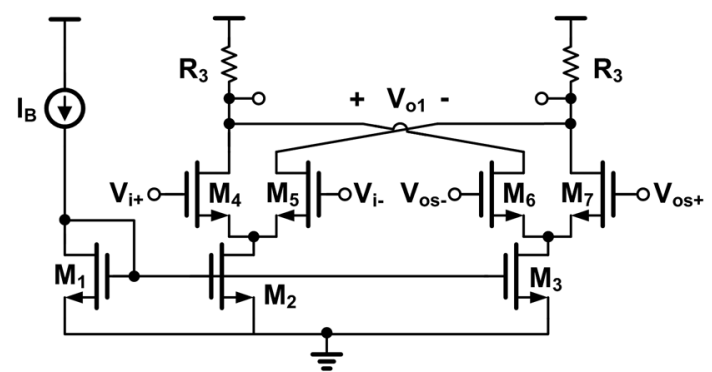

(a)

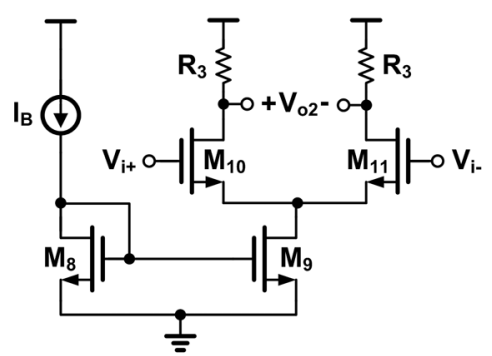

(b)

Fig. 10. (a) Offset-cancellation amplifier (b) gain cell.

which leads to a higher nonlinearity of the single transistor mixer. With on chip VCO amplitude of about $100 \mathrm{mV}$ and tripler output amplitude of about $250 \mathrm{mV}$, the simulated conversion gain of the third harmonic mixer is about $-28 \mathrm{~dB}$.

\section{Post-Amplifier and RSSI}

Fig. 9 shows the post-amplifier architecture. It is composed of an offset cancellation input stage $\left(A_{1}\right)$ followed by five identical gain cells $\left(A_{2}\right)$ and an $\left(R_{1}, C_{1}\right)$ low pass filter for offset extraction. The offset cancellation stage and the gain cells are basically common source amplifiers, as are shown in Fig. 10(a) and Fig. 10(b) respectively. The post amplifier provides a DC gain of $60 \mathrm{~dB}$, and $-3 \mathrm{~dB}$ bandwidth of about $1 \mathrm{GHz}\left(\sim M f_{R E F}\right)$. If $f_{I F}$ falls within the $-3 \mathrm{~dB}$ bandwidth of the post amplifier, it would be enlarged to be higher than $324 \mathrm{mVpp}$ to drive the divider for phase and frequency comparison.

The post amplifier output is connected to a full wave rectifier to perform as received signal strength indicator (RSSI). Fig. 11 shows the detailed circuit schematic. The output voltage is con- 


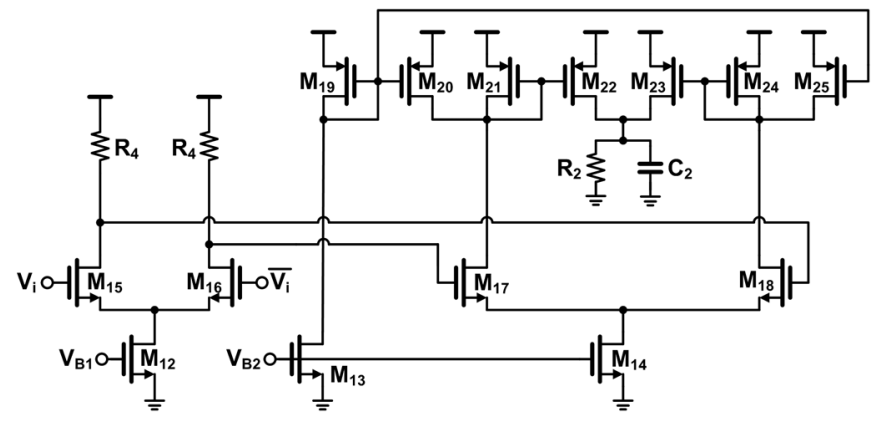

Fig. 11. Full wave rectifier for RSSI.

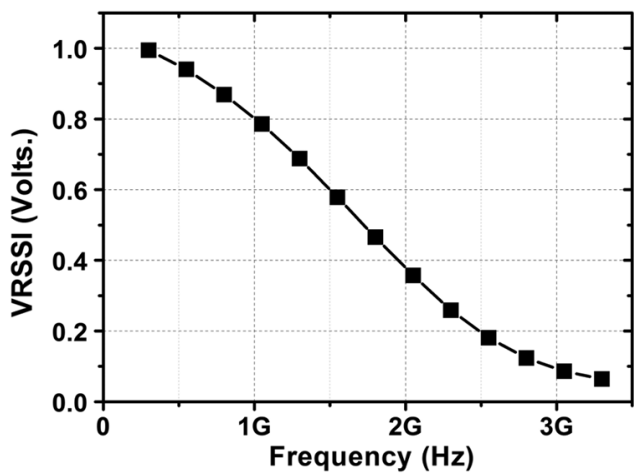

Fig. 12. Simulated frequency dependent output voltage characteristic of RSSI.

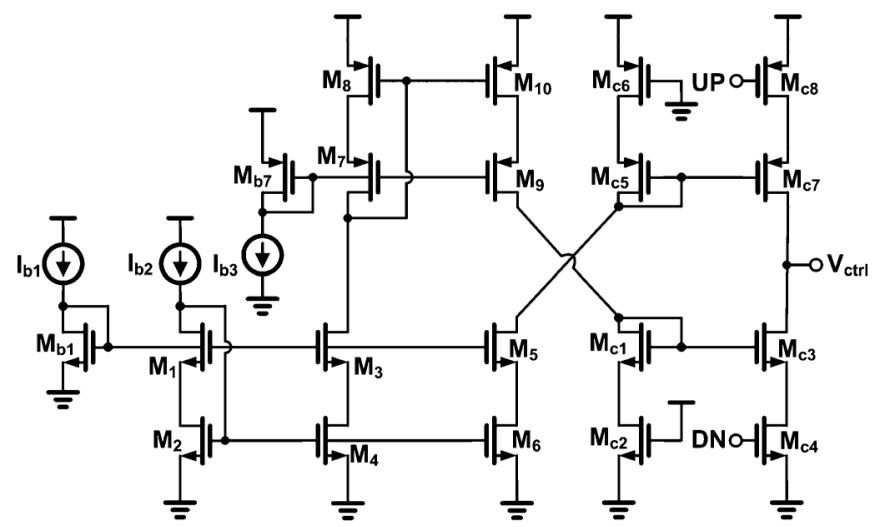

Fig. 13. Charge pump schematic.

verted to current form by $\mathrm{M}_{17}$ and $\mathrm{M}_{18}$, and rectified through current mirrors $\left(\mathrm{M}_{21}-\mathrm{M}_{22}\right)$ and $\left(\mathrm{M}_{23}-\mathrm{M}_{24}\right)$. The RSSI manifests pseudo linear frequency dependent transfer characteristic, as is shown in Fig. 12. It fully covers the tuning range of the VCO to assist frequency pull-in process.

At the onset of frequency lock-in process, the $\mathrm{VCO}$ is preset to its highest frequency, leading to a smaller output at the RSSI. The VCO frequency is then pulled down gradually by a resistorladder DAC, which is controlled by the RSSI. As RSSI exceeds the threshold level, the post amplifier output is large enough to make the succeeding dividers function properly. The PLL will resume its close loop operation for frequency locked and phase tracking.

\section{E. Charge Pump}

Fig. 13 shows the charge pump circuit. To alleviate reference spurs induced by current mismatch, wide swing cascode current

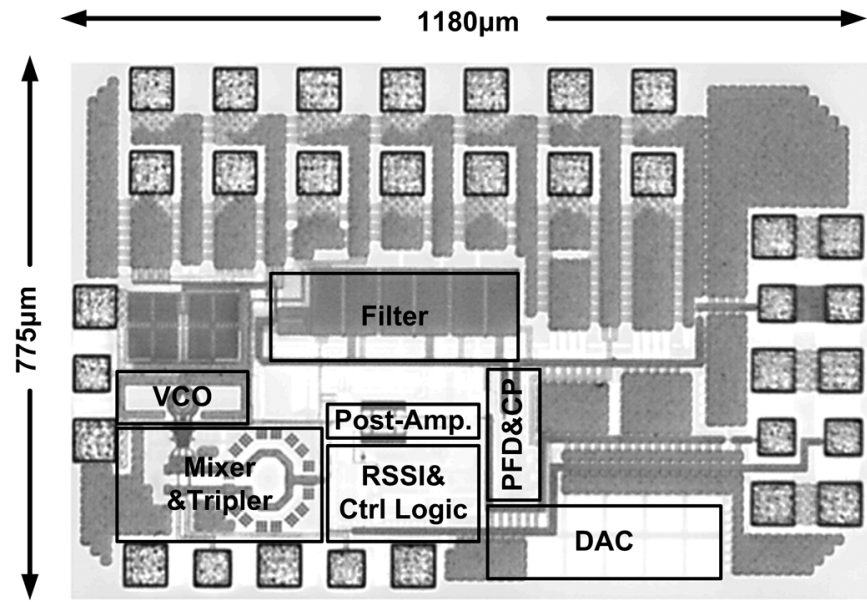

Fig. 14. Chip photograph.

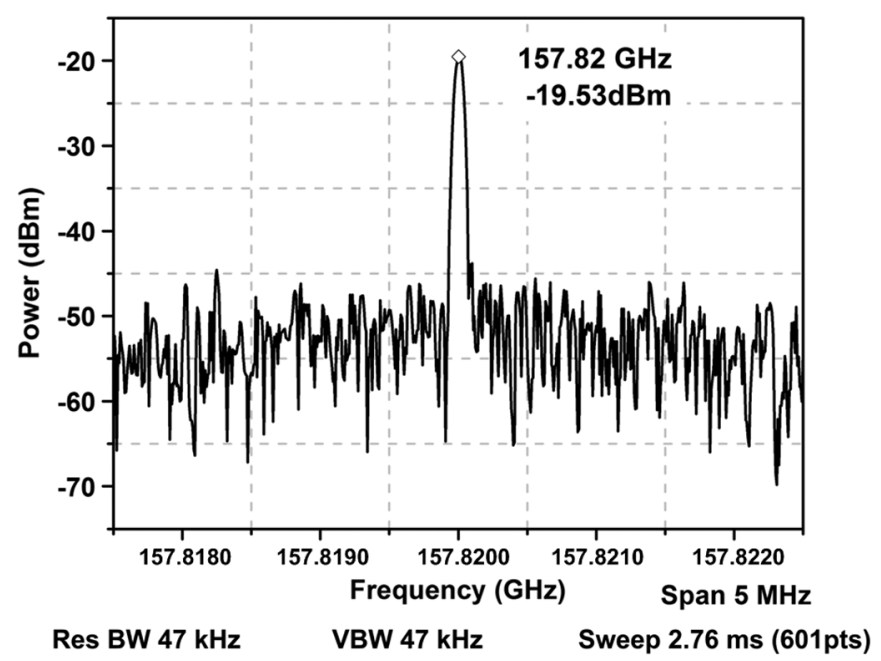

Fig. 15. Measured PLL output spectrum at $157.82 \mathrm{GHz}$ with $f_{R E F}=$ $130 \mathrm{MHz}$.

mirrors are adopted to copy the up and down currents. Here $\mathrm{M}_{\mathrm{c} 2}$ and $\mathrm{M}_{\mathrm{c} 6}$ are replicas of $\mathrm{M}_{\mathrm{c} 4}$ and $\mathrm{M}_{\mathrm{c} 8}$.

\section{EXPERIMEANTAL RESULTS}

A $160 \mathrm{GHz}$ frequency-translation PLL has been fabricated using TSMC $65 \mathrm{~nm}$ CMOS process, and is powered under a $1.2 \mathrm{~V}$ supply. The total power dissipation is about $24 \mathrm{~mW}$ and the chip size is about $775 \mu \mathrm{m} \times 1180 \mu \mathrm{m}$, excluding the frequency multiplier. The chip photo is shown in Fig. 14. The chip performance is measured using probe station. The VCO output frequency is down converted by an external harmonic mixer (OML M05HWD) and measured by frequency spectrum analyzer (Agilent E4448A). With a reference frequency $\left(f_{R E F}\right)$ of $130 \mathrm{MHz}, f_{M}$ at $17.42 \mathrm{GHz}(\mathrm{N}=134)$, and $\mathrm{M}=8$, the measured output spectrum is shown in Fig. 15. By taking the insertion loss $(\sim-55 \mathrm{~dB})$ of external mixer and cable into account, the output power of VCO is $-19 \mathrm{dBm}$. The measured locking time using signal source analyzer (Agilent E5052A) is less than $3 \mu \mathrm{s}$ by the proposed frequency acquisition scheme, as is shown in Fig. 16.

Due to the limitation of in house instruments, the VCO phase noise performance is indirectly measured at the post amplifier 
TABLE I

PERFORMANCE BENCHMARK

\begin{tabular}{|c|c|c|c|c|}
\hline Reference & {$[7]$} & {$[16]$} & {$[17]$} & This work \\
\hline Technology & $65 \mathrm{~nm}$ CMOS & $\begin{array}{c}0.13 \mu \mathrm{m} \\
\text { SiGe BiCMOS }\end{array}$ & InP HBT & $65 \mathrm{~nm}$ CMOS \\
\hline $\mathrm{f}_{\text {out }}(\mathrm{GHz})$ & 104 & 95 & 300 & 158 \\
\hline $\begin{array}{l}\text { Locking Range } \\
\qquad(\mathrm{GHz})\end{array}$ & 1.5 & 7.5 & 0.36 & 2.8 \\
\hline Prescaler & ILFD & Miller Divider & Miller Divider & $\begin{array}{c}\text { Harmonic Mixer } \\
+ \text { RSSI Frequency Sweeping }\end{array}$ \\
\hline$P_{\text {out }}(\mathrm{dBm})$ & -23 & 3 & -23 & -19 \\
\hline Phase noise & $\begin{array}{l}-80.4 \mathrm{dBc} / \mathrm{Hz} \\
\text { (a) } 1 \mathrm{MHz} \text { offset }\end{array}$ & $\begin{array}{l}-102 \mathrm{dBc} / \mathrm{Hz} \\
\text { (a) } 1 \mathrm{MHz} \text { offset }\end{array}$ & $\begin{array}{c}-85 \mathrm{dBc} / \mathrm{Hz} \\
\text { (a) } 1 \mathrm{MHz} \text { offset }\end{array}$ & $\begin{array}{l}-85 \mathrm{dBc} / \mathrm{Hz} \\
\text { (a) } 1 \mathrm{MHz} \text { offset }\end{array}$ \\
\hline Power Dissipation & $63 \mathrm{~mW}$ & $570 \mathrm{~mW}$ & $301.6 \mathrm{~mW}$ & $\begin{array}{c}24 \mathrm{~mW} \\
\text { (w/o frequency multiplier) }\end{array}$ \\
\hline Chip size & $1.1 \times 0.76 \mathrm{~mm}^{2}$ & $1.1 \times 1.1 \mathrm{~mm}^{2}$ & $0.84 \mathrm{~mm}^{2}$ & $\begin{array}{c}1.2 \times 0.8 \mathrm{~mm}^{2} \\
(\mathrm{w} / \mathrm{o} \text { frequency multiplier) }\end{array}$ \\
\hline
\end{tabular}

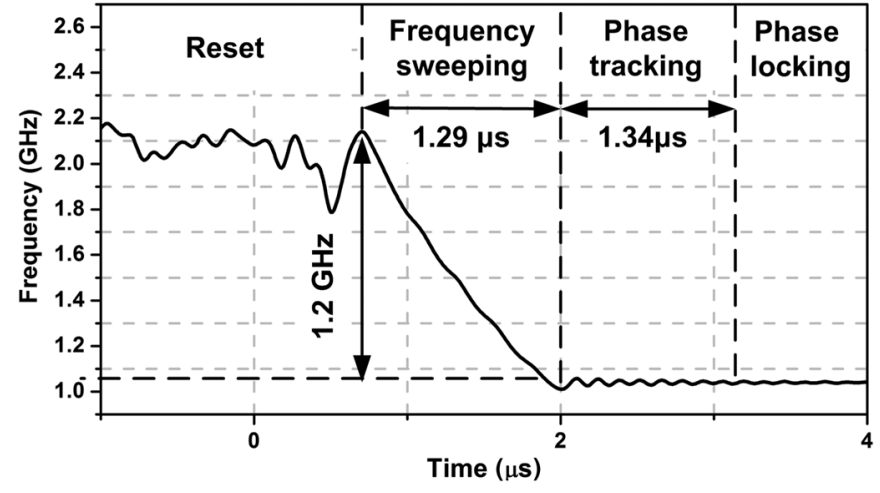

Fig. 16. Measured PLL settling behavior at $f_{I F 2}$ (For $f_{V C O}$ hopping from $158.92 \mathrm{GHz}$ to $157.82 \mathrm{GHz}, f_{I F_{2}}$ changes from $2.14 \mathrm{GHz}$ to $1.04 \mathrm{GHz}$ ).

output $\left(f_{I F 2}\right)$. Fig. 17 shows the measured phase noise performance. According to the previous discussions in Section III, the phase noise performance at amplifier output $\left(\phi_{n, I F 2}\right)$ can be modeled as

$$
\overline{\phi_{n, I F 2}^{2}}=\overline{\phi_{n, V C O}^{2}}+\overline{\phi_{n, \text { excess }}^{2}} \text {. }
$$

By taking the noise contribution of $\phi_{n, \text { excess }}$ into account, the VCO phase noise is expected to be better than $-85 \mathrm{dBc} / \mathrm{Hz}$ at $1 \mathrm{MHz}$ offset.

Table I summaries the performance benchmark. It can be seen that the resonator based divider (ILFD/Miller) are facing severe design challenges as operating frequency beyond $100 \mathrm{GHz}$. Taking the estimated power of auxiliary PLL into account [15],

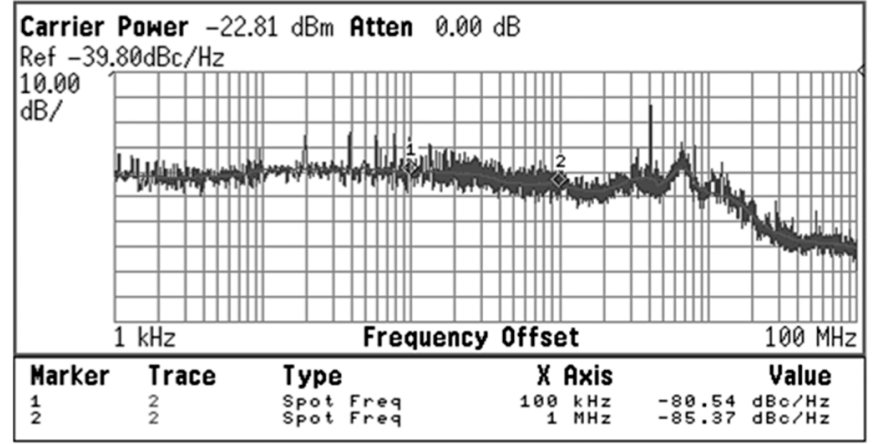

Fig. 17. Measured phase noise performance at $f_{I F 2}$.

the proposed FT-PLL architecture still manifests superiority in low power consumption by circumventing 1/9 millimeter wave prescaler. Additionally, by RSSI assisted automatic frequency sweeping, it can achieve reliable operation without external tuning.

\section{CONCLUSION}

This paper proposes a novel frequency-translation PLL for sub-THz systems. High frequency prescaler in the PLL is replaced by a 3rd harmonic mixer for frequency down conversion. In contrast to conventional PLL with cascaded ILFDs as prescaler, it is free of frequency misalignment problems with the aid of RSSI for automatic frequency sweeping. Finally, a $160 \mathrm{GHz}$ fundamental mode PLL with agile locking speed is demonstrated using TSMC $65 \mathrm{~nm}$ CMOS process. 


\section{REFERENCES}

[1] H. Sherry et al., "A 1 kpixel CMOS camera chip for 25 fps real-time terahertz imaging applications," in Proc. ISSCC Dig. Tech. Papers, Feb. 2012, pp. 252-254.

[2] K. Sengupta and A. Hajimiri, "A $0.28 \mathrm{THz} 4 \times 4$ power-generating and beam-steering array," in Proc. ISSCC Dig. Tech. Papers, Feb. 2012, pp. 256-258.

[3] D. Huang et al., " $324 \mathrm{GHz}$ CMOS frequency generator using linear superposition technique," in Proc. ISSCC Dig. Tech. Papers, Feb. 2008, pp. 476-629.

[4] E. Seok et al., "A $410 \mathrm{GHz}$ CMOS push-push oscillator with an on-chip patch antenna," in Proc. ISSCC Dig. Tech. Papers, Feb. 2008, pp. 472-629.

[5] J. Lee, "A 75-GHz PLL in 90-nm CMOS technology," in Proc. ISSCC Dig. Tech. Papers, Feb. 2007, pp. 432-613.

[6] K.-H. Tsai and S.-I. Liu, "A $43.7 \mathrm{~mW} 96 \mathrm{GHz}$ PLL in $65 \mathrm{~nm}$ CMOS," in Proc. ISSCC Dig. Tech. Papers, Feb. 2009, pp. 276-277.

[7] K.-H. Tsai and S.-I. Liu, "A 104-GHz phase-locked loop using a VCO at second pole frequency," IEEE Trans. Very Large Scale Integr. Syst., vol. 20, no. 1, pp. 80-88, Jan. 2012.

[8] J.-M. Hsu, "A 0.18-mm CMOS offset-PLL upconversion modulation loop IC for DCS1800 transmitter," IEEE J. Solid-State Circuits, vol. 38, no. 4, pp. 603-613, Apr. 2003.

[9] B. Razavi, "The role of PLLs in future wireline transmitters," IEEE Trans. Circuits Syst. I, Reg. Papers, vol. 56, no. 8, pp. 1786-1793, Aug. 2009.

[10] L. L. K. Leung, K. W. C. Chui, and H. C. Luong, “A 1 V dual-band VCO using an integrated variable inductor," in ASSCC, Nov. 2005, pp. 273-276.

[11] M.-C. Chen and C.-Y. Wu, "Design and analysis of CMOS subharmonic injection-locked frequency triplers," IEEE Trans. Microw. Theory Tech., vol. 56, no. 8, pp. 1869-1878, Aug. 2008.

[12] B. Razavi, "A study of injection locking and pulling in oscillator," IEEE J. Solid-State Circuits, vol. 39, pp. 1415-1424, Sep. 2004.

[13] Z. Chen and P. Heydari, "An 85-95.2 GHz transformer-based injection-locked frequencyTripIer in $65 \mathrm{~nm}$," in Proc. IEEE Microw. Symp. Digest, May 2010, pp. 776-779.

[14] M.-F. Huang et al., "Analysis and implementation of a CMOS even harmonic mixer with current reuse for heterodyne/direct conversion receivers," IEEE Trans. Circuits Syst., vol. 52, no. 9, pp. 1741-1750, Sep. 2005.

[15] A. W. L. Ng et al., "A 1-V 24-GHz 17.5-mW phase-locked loop in a 0.18-m CMOS process," IEEE J. Solid-State Circuits, vol. 41, no. 6, pp. 1236-1244, June 2006.

[16] S. Kang, J.-C. Chien, and A. M. Niknejad, "A $100 \mathrm{GHz}$ phase-locked loop in $0.13 \mu \mathrm{m}$ SiGe BiCMOS process," in Proc. IEEE Radio Freq. Integrated Circuits Symp., Jun. 2011, pp. 1-4.

[17] M. Seo1 et al., "A $300 \mathrm{GHz}$ PLL in an InP HBT technology," in Proc. IEEE Int. Microw. Symp. Dig., Jun. 2011, pp. 1-4.

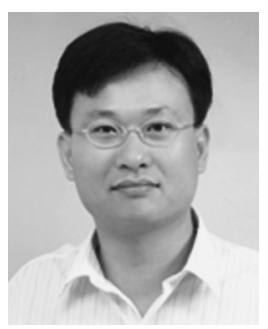

Wei-Zen Chen received the B.S., M.S., and Ph.D. degrees in electronics engineering from National Chiao-Tung University, Hsin-Chu, Taiwan, in 1992, 1994, and 1999, respectively.

He was with ITRI/ERSO in 1999 involved in the development of CMOS RF ICs for cellular and wireless LAN applications. From 1999-2002, he was an Assistant Professor at the Department of Electrical Engineering, National Central University, Chung- $\mathrm{Li}$, Taiwan. Since 2002, he joined the Department of Electronics Engineering, National Chiao-Tung University, where he is now a full Professor. His research focuses on mixed-signal integrated circuit for wireless and wireline communication systems, with special emphasis on Serdes, high speed interface, optical communication, wireless PAN, LAN, and body area network applications.

Dr. Chen was the deputy executive director of National SoC (NSoC) Program in Taiwan from 2009-2011, and is the principal investigator of National Project on Intelligent Electronics (NPIE) in Taiwan since 2011. He also served as IEEE
Solid-State Circuit Society Taipei Chapter chairman from 2008-2012. He was the technical program vice chair of $2008 \mathrm{VLSI} / \mathrm{CAD}$ design symposium, technical program chair of 2012 symposium on engineering, medical, and biology applications (SEMBA), and the guest editor of International Journal of Electrical Engineering (IJEE). He is a member of Phi-Tau-Phi honorary scholar society, and also served as technical program committee member of IEEE Custom Integrated Circuits Conference (CICC), IEEE Asian Solid-State Circuit Conference (A-SSCC), IEEE RFIT, and IEEE ASICON.

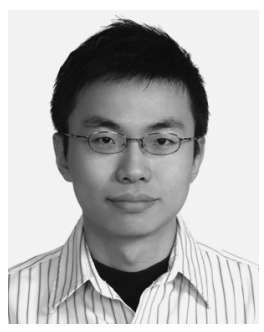

Tai-You Lu was born in Taipei, Taiwan, in 1980. He received the B.S. degree in electrical engineering from National Cheng-Kung University, Tainan, Taiwan, in 2003, and the Ph.D. degree in electronics engineering from National Chiao Tung University, Hsinchu, Taiwan, in 2011.

He has been with MediaTek Inc., Taiwan, since 2011 , where he is working on mixed-signal integrated circuit design for communication systems.

Dr. Lu is a member of Phi Tau Phi honorary scholar society.

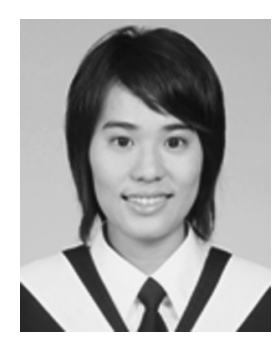

Yan-Ting Wang was born in Chiayi, Taiwan, in 1986. She received the B.S. degree in electrical control engineering from National Chiao-Tung University, Taiwan, in 2009, and the M.S. degree in the Institute of Electronics, National Chiao-Tung University, Hsinchu, Taiwan, in 2012.

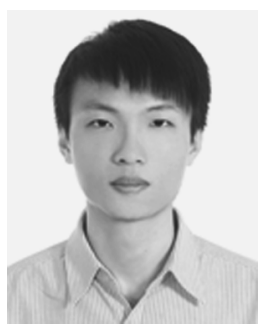

Jhong-Ting Jian was born in Taipei, Taiwan, on April 8, 1987. He received the B.S. degree in electrical engineering from National Sun Yat-Sen University, Kaohsiung, Taiwan, in 2009 and M.S. degree in electronic engineering from National Chiao-Tung University, Hsinchu, Taiwan, in 2012.

He subsequently joined the LTPS department of Sitronix Technology Corporation, Taipei, Taiwan. Since then he has been engaged in the research and development of CMOS mixed-signal circuit for high resolution display driver IC.

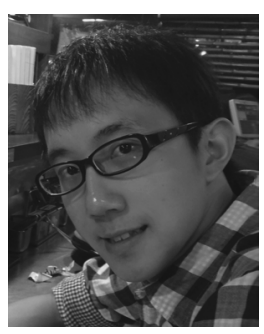

Yi-Hung Yang was born in Taipei, Taiwan, on June 19, 1987.He received the B.S. degree in the Department of Electrical Engineering from National Sun Yat-Sen University, Taiwan, and the M.S. degree in the Institute of Electronics from National Chiao-Tung University, Taiwan, in 2009 and 2012, respectively.

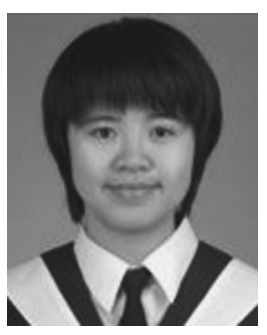

Kai-Ting Chang was born in Taichung, Taiwan, in 1988. She received the B.S. degree in electrical engineering from National Central University, Taiwan, in 2011. She is currently working toward the M.S. degree at National Chiao-Tung University, Hsinchu, Taiwan. 